J. Dairy Sci. 96:3525-3534

http://dx.doi.org/10.3168/jds.2012-6514

(C) American Dairy Science Association ${ }^{\circledR}, 2013$.

\title{
Oral administration of Lactobacillus delbrueckii subspecies bulgaricus OLL1073R-1 suppresses inflammation by decreasing interleukin- 6 responses in a murine model of atopic dermatitis
}

\author{
Hiroshi Kano, ${ }^{1}$ Junko Kita, Seiya Makino, Shuji Ikegami, and Hiroyuki Itoh \\ Food Science Research Laboratories, Meiji Corporation, 540 Naruda, Odawara, Kanagawa 250-0862, Japan
}

\begin{abstract}
The oral intake of Lactobacillus spp. can provide beneficial effects to the host by modulating the immune response. Atopic dermatitis (AD) is an allergic inflammatory disease mediated by various immune responses. In this study, we examined the effect of a Lactobacillus strain, Lactobacillus delbrueckii ssp. bulgaricus OLL1073R-1 (OLL1073R-1), on AD development in a murine model of $\mathrm{AD}$ that was developed by the topical application of mite antigen in NC/Nga mice. The oral intake of heat-killed OLL1073R-1 cells inhibited both the development of dermatitis and the elevation of an acute inflammation marker, serum amyloid A. Another bacterial strain, Lactobacillus rhamnosus OLL2984, exerted no inhibitory effects on dermatitis. The oral intake of heat-killed OLL1073R-1 cells also attenuated secretion of IL-6 from lymph node cells in response to mite antigen and reduced IL-6 levels in inflamed tissues, such as auricles. Production of IFN- $\gamma$ or IL-4 was not influenced by OLL1073R-1 intake. We also found that inhibition of IL-6 signaling by gp130-Fc (a fusion protein consisting of the extracellular portion of glycoprotein 130 fused to the Fc region of human IgG1) markedly decreased the severity of dermatitis in NC/Nga mice. Moreover, secretion of IL-6 by lymph node cells was augmented in NC/Nga mice compared with that in BALB/c mice. These results indicate that IL-6 plays an essential role in the development of dermatitis in the NC/Nga mouse model of $\mathrm{AD}$, and that OLL1073R-1 inhibits dermatitis, at least in part, by suppressing the IL-6 response.
\end{abstract}

Key words: atopic dermatitis, Lactobacillus delbrueckii ssp. bulgaricus, interleukin-6, serum amyloid A

\section{INTRODUCTION}

Lactobacilli constitute a part of the intestinal microflora in humans, and are used for production of yogurt

Received December 21, 2012.

Accepted February 19, 2013.

${ }^{1}$ Corresponding author: hiroshi.kano@meiji.com and other dairy and nondairy fermented foods. Many reports have demonstrated that these bacteria provide beneficial effects to the host by modulating the immune response. Some strains of lactobacilli can also decrease the severity of allergic symptoms by modulating effects on Th1/Th2 balance (Hasegawa et al., 2009).

Atopic dermatitis (AD) is a chronic relapsing inflammatory skin disease. A study by Kalliomäki et al. (2001) found that oral administration of a Lactobacillus strain, given prenatally to mothers, was effective in preventing early AD in children at high risk; however, levels of total IgE were similar between lactobacilli-treated and placebo groups. In a later report, the authors suggested that the protective effect of bacterial components on $\mathrm{AD}$ was at least partly IgE-independent (Kalliomäki et al., 2003). Supplementation with a different Lactobacillus strain in children was also shown to be beneficial in reducing the clinical severity of $\mathrm{AD}$, and did not affect the levels of total IgE (Han et al., 2012). Thus, it appears that certain strains of Lactobacillus are able to decrease the severity of $\mathrm{AD}$, and mechanisms other than inhibition of Th2 responses are involved in its amelioration. Atopic dermatitis has characteristics of both allergic and inflammatory responses. Acute AD lesions have a significant increase in the number of cells expressing Th2 cytokines at the mRNA and protein levels, such as IL-4, IL-5, and IL-13; whereas expression of IFN- $\gamma$, the main Th1 cytokine, is predominant in chronic eczematous AD skin lesions (Spergel et al., 1999). In addition, one study showed that peripheral blood mononuclear cells from patients with AD produced significantly higher levels of the proinflammatory cytokine, IL-6, compared with nonatopic controls (Bunikowski et al., 2001). Samochocki et al. (2012) also reported that the serum concentration of IL-6 correlated positively with the severity of $\mathrm{AD}$. These findings indicate that Th1, Th2, and proinflammatory cytokines contribute to the development of $\mathrm{AD}$ depending upon the pathologic stage. Some Lactobacillus strains can downregulate secretion of proinflammatory cytokines from immune cells and intestinal epithelial cells (Chiba et al., 2010; Shimazu et al., 2012). It has also been suggested that ingestion of certain Lactobacillus strains 
can prevent the development of skin legions in an $\mathrm{AD}$ model, probably through induction of the inhibitory cytokine, IL-10, in intestinal lymphoid organs and systemic levels (Sawada et al., 2007). However, it remains unclear whether lactobacilli inhibit the development of dermatitis by reducing proinflammatory cytokines or inflammation markers.

One proinflammatory cytokine, IL-6, is known to have pleiotropic effects on cell growth, differentiation, survival, and migration during immune responses and inflammation via activation of signal transducer and activator of transcription 3 (STAT3; Park et al., 2004; Doganci et al., 2005). A crucial role is played by STAT3 in transducing signals required for keratinocyte migration; likewise, STAT3 are essential for skin remodeling, including the hair cycle and wound healing (Sano et al., 1999). In contrast, transgenic mice with keratinocytes expressing constitutively active STAT3 develop a skin phenotype resembling psoriasis (Sano et al., 2005). It has also been found that STAT3 was consistently activated in epidermal keratinocytes in human psoriatic lesions (Sano et al., 2008). These studies indicate that IL-6 plays important roles in the homeostasis of dermal cells. However, the involvement of IL-6 in the development of $\mathrm{AD}$-like skin lesions in mouse models has yet to be elucidated.

In the present study, we used the $\mathrm{NC} / \mathrm{Nga}$ strain of mice as an in vivo model of $\mathrm{AD}$ to investigate the effects of Lactobacillus delbrueckii ssp. bulgaricus OLL1073R-1 (henceforth referred to by its strain number, OLL1073R-1) on the production of IL-6 and serum amyloid A (SAA), a blood marker of acute inflammation. A previous study demonstrated that this bacterial strain decreased the severity of arthritis in a murine model of rheumatoid arthritis (Kano et al., 2002a). In addition, lymph node cells (LNC) from mice fed a similar bacterial culture secreted less inflammatory mediators, such as IL-6 and tumor necrosis factor- $\alpha$, in a murine arthritis model (Kano et al., 2002b). As $\mathrm{AD}$ is an inflammatory disease, we presumed that OLL1073R-1 would exert inhibitory effects on AD in a murine model. We also confirmed the role of IL-6 in the pathogenesis of dermatitis using $\mathrm{NC}$ /Nga mice. Interleukin-6 acts via a specific receptor complex that consists of a membrane-bound IL-6 or soluble IL-6 receptor and glycoprotein 130 (gp130) (Doganci et al., 2005). Here, we used a fusion protein consisting of the extracellular portion of gp130 fused to the Fc region of human IgG1 (gp130-Fc), and investigated whether the IL-6-soluble IL-6 receptor system contributed to the pathogenesis of AD-like lesions in NC/Nga mice. In addition, we compared the profile of cytokine secretion from LNC of NC/Nga mice with that from another strain of mice.

\section{MATERIALS AND METHODS}

\section{Mice}

Specific-pathogen-free female NC/Nga mice and BALB/c mice, 4- or 5-wk-old, were purchased from Japan SLC (Shizuoka, Japan). Five-week-old mice were used for investigating the effects of lactobacilli and gp130-Fc, whereas 6-wk-old mice were used for comparing immune responses between the 2 mice strains. The experimental protocols were approved by the Ethical Committee for Animal Experiments of Meiji Corporation.

\section{Microorganisms}

We used OLL1073R-1 and Lactobacillus rhamnosus OLL2984 (henceforth referred to by its strain number, OLL2984) in this study. These bacteria were grown in de Man, Rogosa, and Sharpe broth (Becton Dickinson, Sparks, MD) at $37^{\circ} \mathrm{C}$ for $18 \mathrm{~h}$. Bacterial cells were collected by centrifugation at $4^{\circ} \mathrm{C}$ for $15 \mathrm{~min}$ at $8,300 \times$ g. After washing twice with $0.85 \% \mathrm{NaCl}$ followed by one wash with distilled water, the bacterial cells were heat-killed at $75^{\circ} \mathrm{C}$ for $60 \mathrm{~min}$ and lyophilized.

\section{Preparation of Mite Extracts}

Frozen Dermatophagoides pteronyssinus mite bodies (LSL, Tokyo, Japan) were defatted, extracted 1:10 (wt/ vol) with distilled water, and centrifuged. The obtained supernatant (extract) was filtered and lyophilized. After adjusting the protein concentration to $4.5 \mathrm{mg} / \mathrm{mL}$ using the Biorad detergent compatible Protein Assay (Bio-Rad Laboratories, Hercules, CA), the obtained $D$. pteronyssinus extract $(\mathbf{D p})$ was stored at $-80^{\circ} \mathrm{C}$ until subsequent use for induction of dermatitis.

\section{Induction and Assessment of Dermatitis}

After suspension in distilled water, OLL1073R-1 or OLL2984 bacterial cells were orally administered (1 $\mathrm{mg} / \mathrm{d}$ ) to NC/Nga mice throughout the course of the experiment. The control group of mice received distilled water without bacteria. On d 8, after the start of feeding, AD-like skin lesions were induced using a modified version of the method described by Unno et al. (2001). Prepared Dp was applied daily for $14 \mathrm{~d}$ with a brush to the sheared back skin, neck skin, and auricles. Prior to each application, $4 \%$ SDS was applied to disrupt the skin barrier. In the sham group, sterile distilled water was applied instead of Dp. The skin status was observed on $\mathrm{d} 14$ after the beginning of dermatitis induction. The degrees of erythema or hemorrhage, scratch, edema, excoriation or erosion, and scaling or dryness were each 
scored as 0 (none), 1 (mild), 2 (moderate), and 3 points (severe). The sum of these scores was defined as the skin score, as described by Shimizu et al. (2006). When comparing immune responses between $\mathrm{NC} / \mathrm{Nga}$ and $\mathrm{BALB} / \mathrm{c}$ mice, oral administration was not conducted, and induction of dermatitis was the same as previously described.

\section{Measurement of Serum IgE and SAA Levels}

Serum samples were collected on d 15 after the start of Dp application and stored at $-80^{\circ} \mathrm{C}$ for subsequent analysis. Total serum $\operatorname{IgE}$ levels were determined by sandwich ELISA according to Sashihara et al. (2006). Capture antibody, biotin-conjugated antibody, standard $\operatorname{IgE}$, and streptavidin-horseradish conjugate were purchased from BD Biosciences (Franklin Lakes, NJ). The SAA levels in the serum samples were measured using the mouse serum amyloid A ELISA kit (Invitrogen, Carlsbad, CA).

\section{Cytokine Assay}

After blood samples were collected for measurement of $\operatorname{IgE}$ and SAA, mice were euthanized by cervical dislocation, and cervical lymph nodes were removed. Lymph node cells were prepared and cultured $(2.5 \times$ $10^{6}$ cells $/ \mathrm{mL}$ ) with $10 \mu \mathrm{g} / \mathrm{mL}$ of sterilized Dp or immobilized anti-CD3 antibodies (BD Biosciences) in RPMI 1640 medium (Invitrogen). The medium contained 10\% fetal calf serum (Intergen, Purchase, NY), $100 \mathrm{U} / \mathrm{mL}$ of penicillin, $100 \mu \mathrm{g} / \mathrm{mL}$ of streptomycin, $0.05 \mathrm{mM}$ of 2-mercaptoethanol, $2 \mathrm{~m} M$ of L-glutamate, $1 \mathrm{mM}$ of sodium pyruvate, and $0.1 \mathrm{~m} M$ of nonessential AA. After $48 \mathrm{~h}$ of incubation, supernatants were collected for measurement of various cytokine levels. After the mice were euthanized, the auricles were removed and protein was extracted using a cell lysis kit (Bio-Rad) for measurement of cytokine levels. The ELISA kits from R\&D systems (Minneapolis, MN) were used for measuring levels of IL-13, and BD OptEIA ELISA Sets (BD Biosciences) were used for measuring levels of IL4, IL-6, and IFN- $\gamma$.

\section{Inhibition of Responses to IL-6 by gp130-Fc}

Dermatitis was induced in NC/Nga mice for $14 \mathrm{~d}$ as described above. For $7 \mathrm{~d}$ before initiation of dermatitis and $1 \mathrm{~h}$ before application of $\mathrm{Dp}, 5 \mu \mathrm{g} /$ mouse of gp130-Fc (R\&D Systems) in PBS or vehicle was injected intracutaneously to the neck of each mouse. The dermatitis score and levels of $\mathrm{IgE}$ and SAA were evaluated as described above.

\section{Protein Extraction and Western Blot Analysis}

The auricle protein extracts were prepared as described in the Cytokine Assay section, and SDS sample buffer was added to equal amounts of protein. After boiling, proteins $(7.5 \mu \mathrm{g})$ were resolved on 8 to $16 \%$ SDS-PAGE gels (Cosmo Bio, Tokyo, Japan), and transferred to Immobilon-P membranes (Merck KGaA, Darmstadt, Germany). After blocking, the membranes were immunoblotted with antibodies specific to the following target proteins: STAT1, phospho (p) STAT1 (BD Biosciences), STAT3, pSTAT3, STAT6 (Cell Signaling Technology, Danvers, MA), and pSTAT6 (IMGENEX, San Diego, CA). This was followed by washing and incubation with peroxidase-conjugated antibodies. The STAT proteins were detected with Immobilon Western Chemiluminescent HRP Substrate (Merck KGaA).

\section{Statistical Analyses}

Significance of differences between the experimental and control groups was examined by the Student's $t$-test or ANOVA employing the Dunnett's post hoc test. Any $P$-values $<0.05$ were considered statistically significant.

\section{RESULTS}

\section{Inhibitory Effect of OLL1073R-1 on the Development of Dermatitis}

We compared the dermatitis scores, IgE levels, and SAA levels in groups treated with heat-killed OLL1073R-1, heat-killed OLL2984, or water. We conducted several analyses by using samples obtained from mice on d 15 after induction of dermatitis, because we found a remarkable difference between each group at that time point. The dermatitis score, but not IgE levels, decreased in the OLL1073R-1-treated group (Figure 1). However, no decrease in the dermatitis score or IgE levels was observed in the OLL2984-treated group compared with the control group. The SAA levels were markedly elevated when dermatitis was induced by application with Dp compared with the sham group (Figure 1). In addition, similar to what was observed with the dermatitis score, SAA levels decreased in the OLL1073R-1-treated group, but not in the OLL2984treated group (Figure 1).

\section{Downregulation of IL-6 Responses by OLL1073R-1}

To investigate the mechanisms contributing to inhibition of dermatitis by heat-killed OLL1073R-1, we analyzed cytokine levels in the inflamed tissue of the 


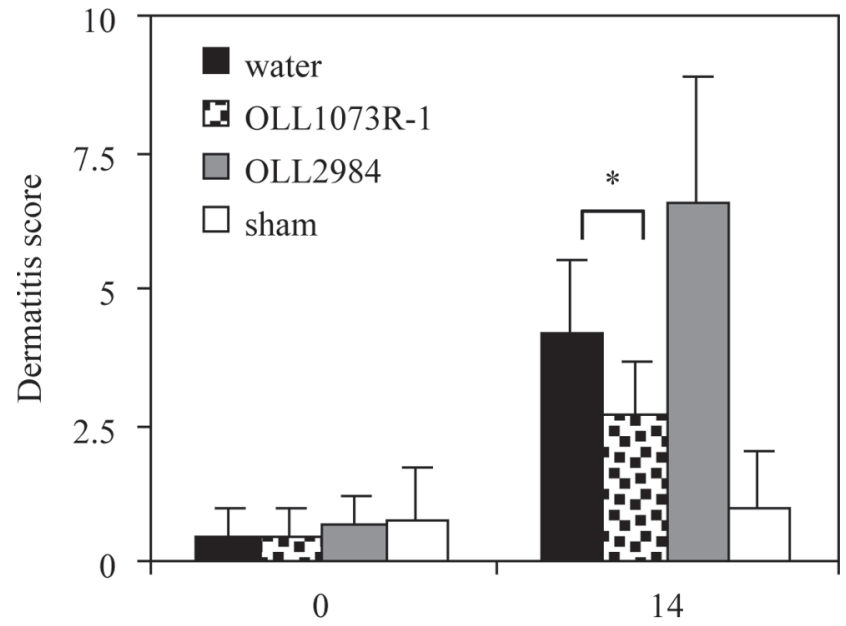

Days after the start of Dp-application

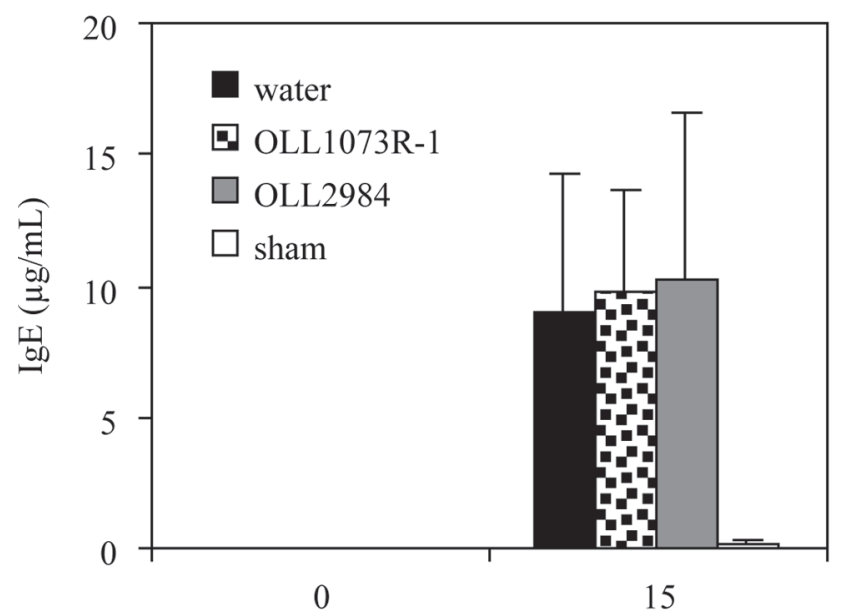

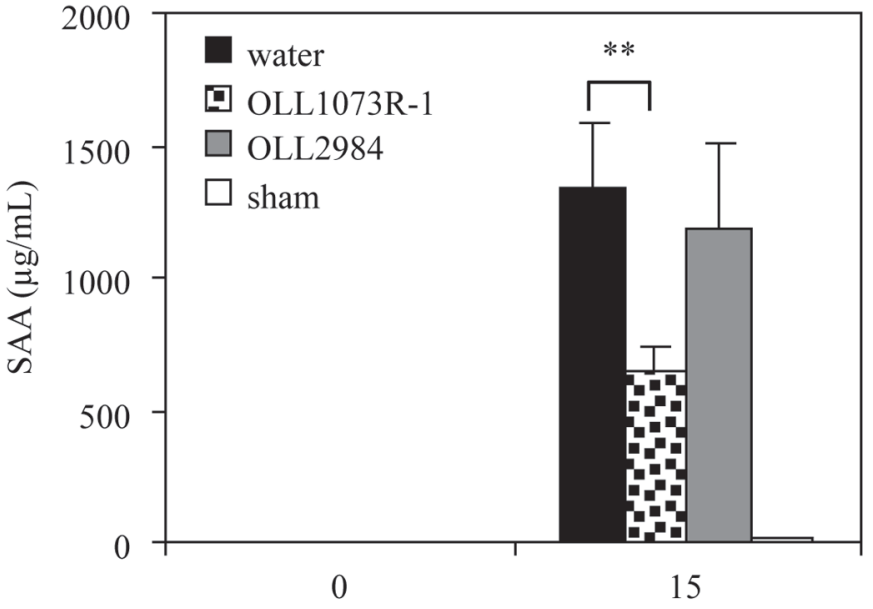

Days after the start of Dp-application

Days after the start of Dp-application

Figure 1. Effect of Lactobacillus delbrueckii ssp. bulgaricus OLL1073R-1 (OLL1073R-1) on dermatitis scores, total serum IgE levels, and serum amyloid A (SAA) levels in NC/Nga mice. Mice were fed OLL1073R-1, Lactobacillus rhamnosus OLL2984 (OLL2984), or water for 7 d, after which SDS and Dermatophagoides pteronyssinus extract (dermatitis-induced groups) or SDS and water (sham group) were applied for 14 d. Administration with lactobacilli was continued during the period of dermatitis induction. Data are presented as mean \pm SD of 6 mice in each group. ${ }^{*} P<0.05$ and ${ }^{* *} P<0.01$ compared with the control group using the Dunnett's test.

auricle, as well as cytokine production by LNC cultured with Dp. The IL-6 levels were lower in the OLL1073R1-treated group, but not in the OLL2984-treated group, compared with the control group (Figure 2A). Levels of IFN- $\gamma$ decreased in dermatitis-induced groups, and little difference was found between groups regardless of bacterial treatment. The IL-13 levels increased upon induction of dermatitis, and oral administration of OLL1073R-1 did not inhibit IL-13 elevation with statistical significance. Expression of IL-4 was not detected in any group (data not shown).

Secretion of IL- 6 by LNC was attenuated in mice treated with OLL1073R-1 compared with mice in the control group (Figure 2B); however, administration of
OLL2984 had no effect on IL-6 production. In addition, no statistically significant decrease in the secretion of the other cytokines tested was noted, which included IFN- $\gamma$, IL-4, or IL-13, in the OLL1073R-1 or OLL2984 treatment groups.

\section{Comparison of Sensitivity to Dermatitis Induction and Cytokine Responses Between NC/Nga and BALB/C Mice}

To determine the key factor in the development of dermatitis in this AD model, we compared dermatitis scores as well as IgE and SAA levels between NC/ $\mathrm{Nga}$ and BALB/c mice whether or not dermatitis was 
A
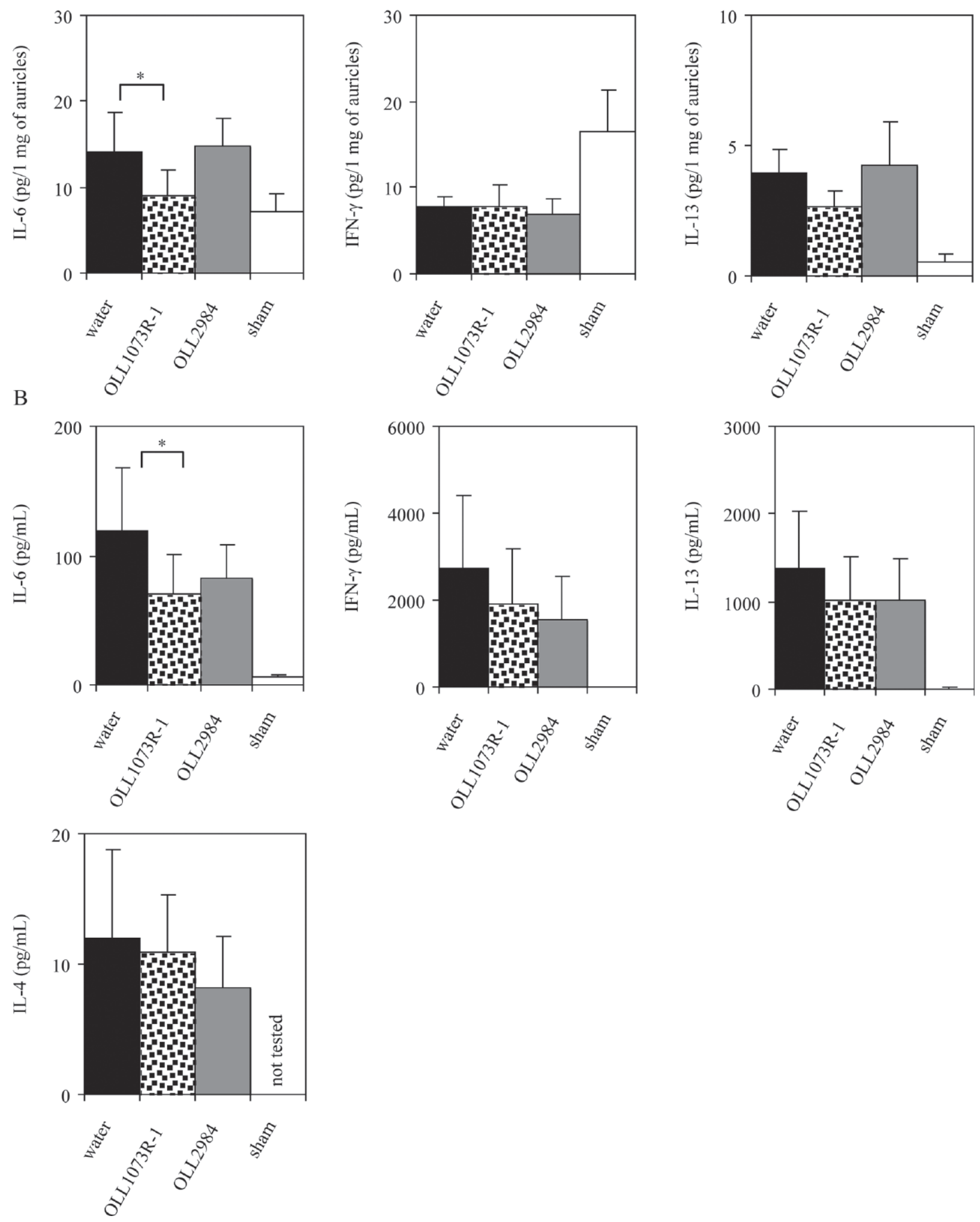

Figure 2. Cytokine levels in the auricles (A) and cytokine production by cervical lymph node cells (B) from the atopic dermatitis mouse model that was orally administered Lactobacillus strains. Experimental designs are the same as described in Figure 1. Fifteen days after the beginning of Dermatophagoides pteronyssinus extract application to the mice, auricles and lymph node cells were collected and used for analyses. Data represent mean $\pm \mathrm{SD}$ of 6 mice in each group. ${ }^{*} P<0.05$ compared with the control group using the Dunnett's test. 
induced. Dermatitis scores and SAA levels were much higher in NC/Nga than BALB/c mice upon application with Dp (Figure 3A). In contrast, IgE levels in $\mathrm{BALB} / \mathrm{c}$ mice were the same or more than that in $\mathrm{NC} /$ Nga mice whether or not dermatitis was induced by Dp application.

We investigated cytokine secretion by LNC cultured in the presence of immobilized anti-CD3 antibodies from $\mathrm{NC} / \mathrm{Nga}$ and $\mathrm{BALB} / \mathrm{c}$ mice whether or not dermatitis was induced. More IL-6 was produced by LNC from $\mathrm{NC} / \mathrm{Nga}$ than $\mathrm{BALB} / \mathrm{c}$ mice whether or not dermatitis was induced by Dp application (Figure 3B). In contrast, LNC from BALB/c mice produced more IL-4 and IL-13 than that from NC/Nga mice. No significant difference was found for IL-13 production in dermatitisinduced mice. More IFN- $\gamma$ was produced by LNC from NC/Nga mice applied with Dp than BALB/c mice.

\section{Inhibitory Effect of gp130-Fc on the Development of Dermatitis}

We investigated the effect of gp130-Fc, which inhibits IL-6 signaling, on the development of dermatitis in $\mathrm{NC} / \mathrm{Nga}$ mice. Intradermal administration of gp130-Fc markedly reduced the severity of skin inflammation and SAA levels, but not IgE levels (Figure 4A). We confirmed the inhibitory effects of gp130-Fc on the IL-6 response by examining pSTAT3 expression in the auricles, and found an increase in pSTAT3 expression in the dermatitis-induced groups compared with the sham group (Figure 4B). The expression level of pSTAT3 was attenuated in the gp130-Fc-treated group compared with the control group. The expression levels of pSTAT1 also increased upon dermatitis induction, but were similar between the gp130-Fc-treated and control groups (Figure 4B). Regardless of its phosphorylation state, STAT6 expression was diminished upon dermatitis induction; however, this effect was reversed with gp130-Fc treatment.

\section{DISCUSSION}

In this study, we investigated whether OLL1073R-1 was able to suppress the development of allergic inflammation. Oral administration of heat-killed OLL1073R-1 cells decreased the severity of dermatitis in an AD murine model. This inhibitory effect on skin inflammation was dependent upon the type of bacterial species or strain, because the OLL2984 bacterial strain did not suppress skin inflammation or cytokine production. Although OLL1073R-1 inhibited the development of dermatitis, these bacteria had little effect on IgE levels, indicating that the inhibitory effect of OLL1073R-1 on dermatitis is not merely mediated by suppression of Th2 responses. Immunoglobulin E plays an essential role in allergic responses, and most patients have increased $\operatorname{IgE}$ serum levels against several allergens (Wüthrich, 1978). However, a decrease in IgE levels is not necessarily accompanied by amelioration of $\mathrm{AD}$. Lactobacillus sakei LK-117 also reduced the development of AD-like skin lesions in a manner independent of the IgE plasma levels in the NC/Nga mouse model (Masuda et al., 2012). Thus, OLL1073R-1 might partially share a similar mechanism with bacterial strain of LK-117. Here, we found a remarkable increase in SAA levels in NC/Nga mice with skin inflammation, and treatment with heat-killed OLL1073R-1 cells inhibited this increase. The increase in SAA levels paralleled that of C-reactive protein, which is a major inflammation marker (Chambers et al., 1991). Thus, SAA is likely to be a marker of skin inflammation in this $\mathrm{AD}$ murine model. Interleukin-6 induced SAA synthesis in a hepatoma cell line (Ganapathi et al., 1988). Reduced levels of SAA by OLL1073R-1 may result from attenuated production of IL-6.

In a previous study, a culture of OLL1073R-1 markedly inhibited the development of arthritis, whereas bacterial cells of that strain did not exhibit inhibitory effects (Kano et al., 2002a). In contrast, heat-killed bacterial cells of that strain decreased the severity of AD-like skin lesions in this study. This difference may be the result of different experimental conditions, such as animal models, antigens, and the timing of feeding of bacterial cells. For example, in this study, we treated mice with heat-killed OLL1073R-1 cells, even after induction of skin inflammation, whereas in the previous study, mice were treated with OLL1073R-1 only before the induction of arthritis. Thus, the effect of OLL1073R-1 on different mouse strains, antigens, and route of antigen delivery needs further investigation.

To investigate possible mechanisms by which ADlike inflammation was inhibited by OLL1073R-1, we examined the effect of these heat-killed bacterial cells on the cytokine profile secreted by LNC or inflamed tissues. We found that feeding heat-killed OLL1073R-1 cells particularly inhibited IL-6 production in induced mouse models of inflammation. Clinical data and animal models suggest that IL-6 play critical roles in the pathogenesis of inflammatory diseases, including rheumatoid arthritis, psoriasis, and active Crohn's disease (Ishihara and Hirano, 2002; Goodman et al., 2009; Rincon, 2012). The involvement of IL-6 in the pathogenesis of $\mathrm{AD}$ was also shown in a study that demonstrated IL-6 release in the cutaneous responses to allergen challenges in atopic patients (Lee et al., 1992). Therefore, it is likely that the inhibitory effects of OLL1073R-1 on dermatitis were due to the reduction of IL-6 production in this murine model. 
A
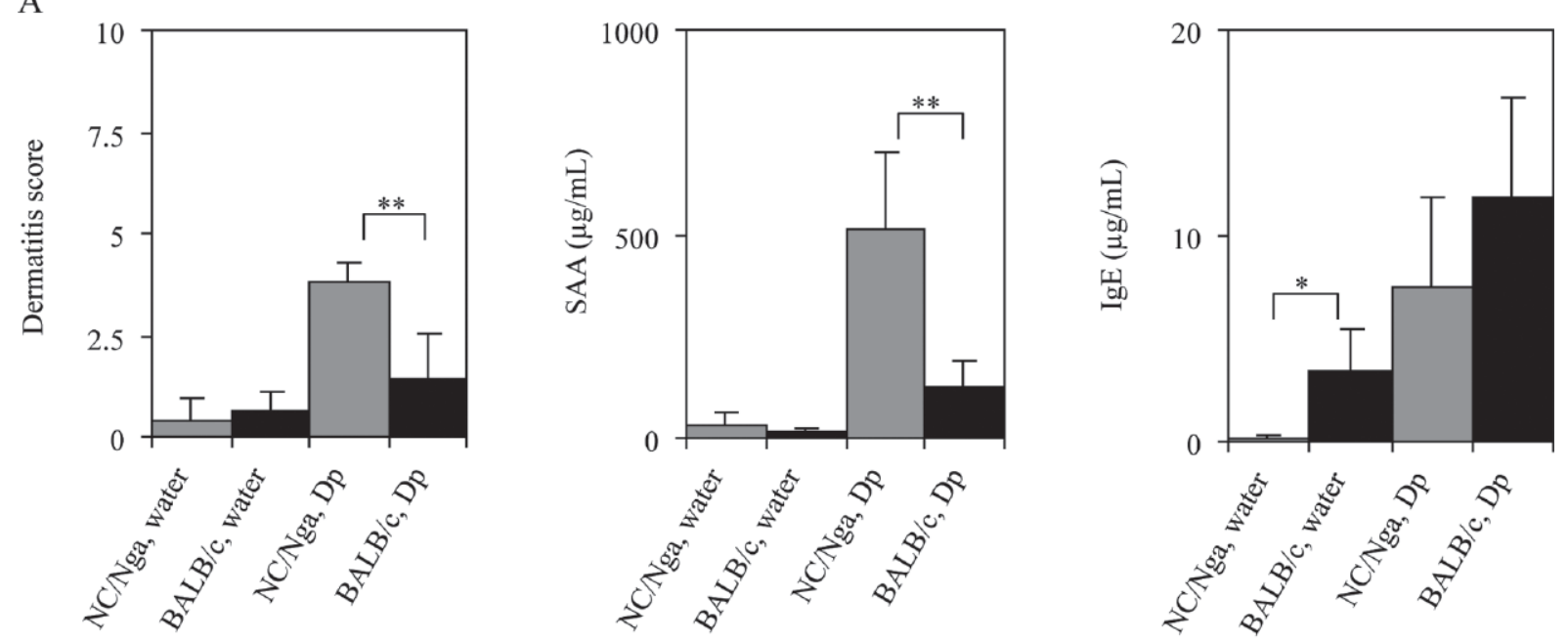

B
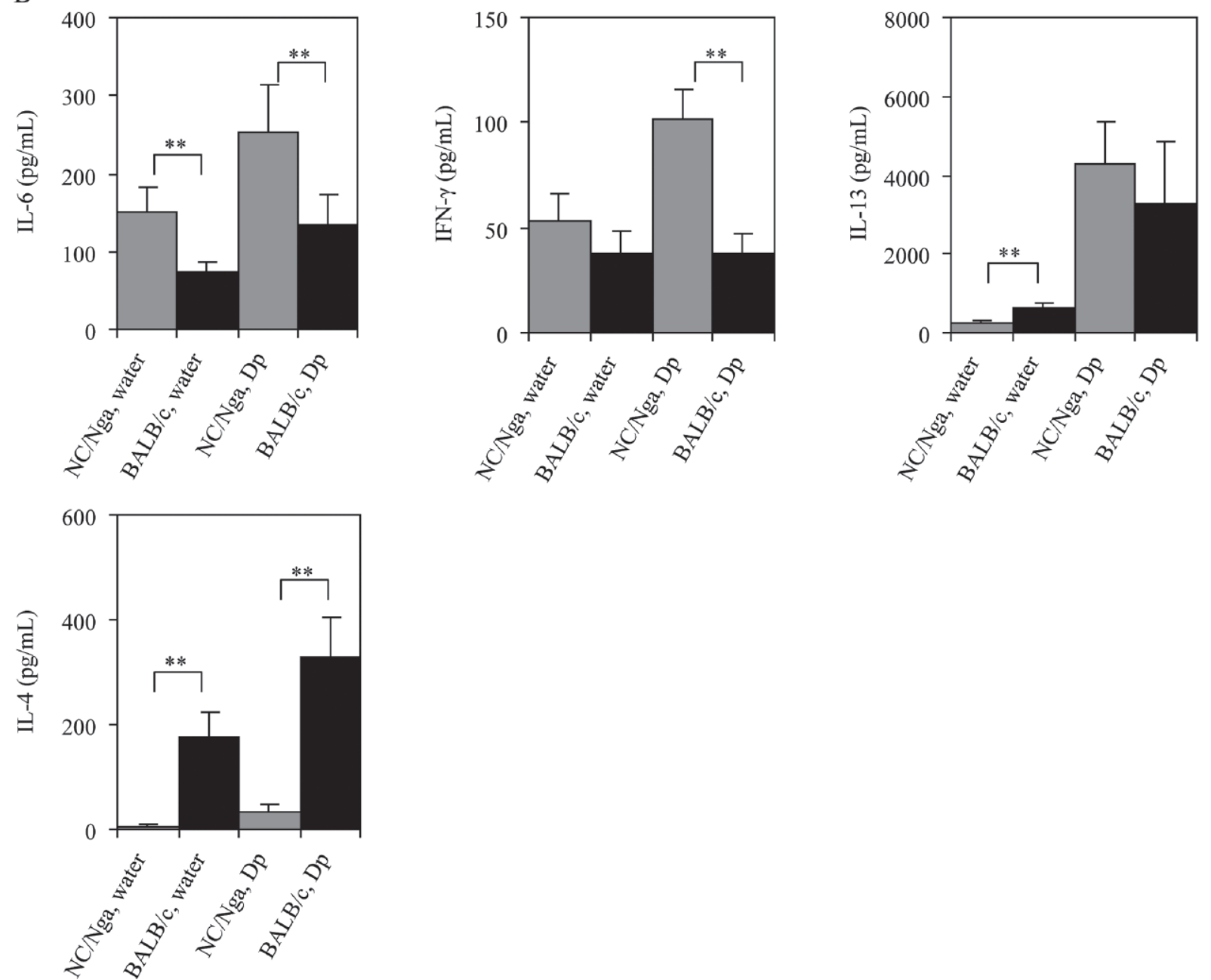

Figure 3. Dermatitis scores, total IgE serum levels, serum amyloid A (SAA) levels (A) and cytokine production by lymph node cells (B) from NC/Nga and BALB/c mice. Sodium dodecyl sulfate and mite extracts, Dermatophagoides pteronyssinus extract (dermatitis-induced groups), or SDS and water (sham group) were applied to mice for $14 \mathrm{~d}$. Other experimental methods are the same as described in Figures 1 and 2 . Data are presented as mean $\pm \mathrm{SD}$ of 5 mice in each group. ${ }^{*} P<0.05$ and ${ }^{* *} P<0.01$ for $\mathrm{NC} / \mathrm{Nga}$ versus BALB/c mice with the same treatment (application with Dp or water) using the Student's $t$-test. 
A

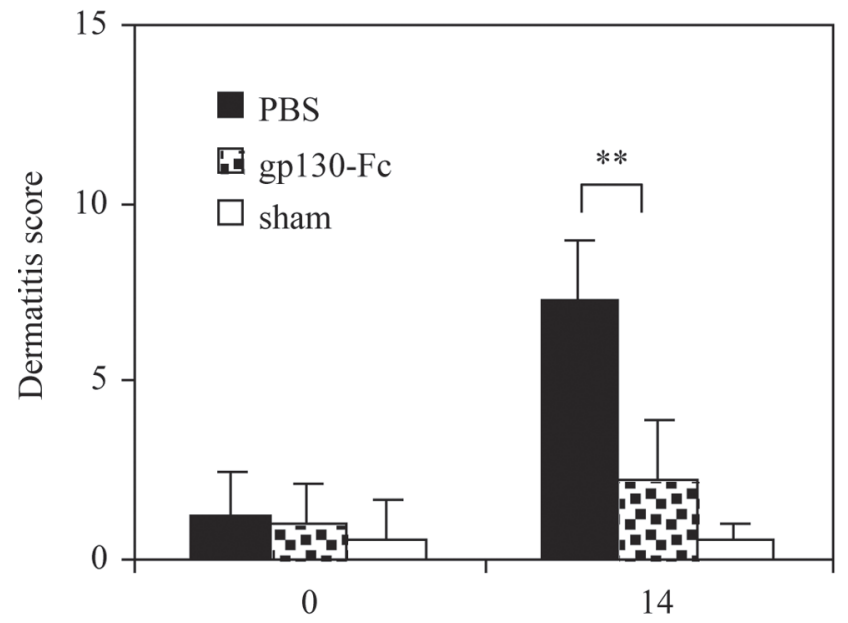

Days after the start of Dp-application

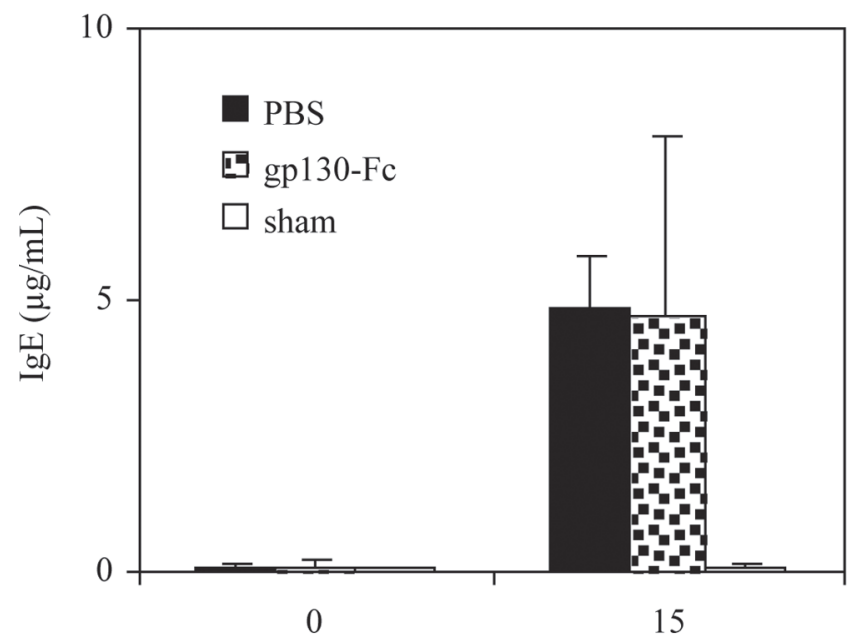

Days after the start of Dp-application

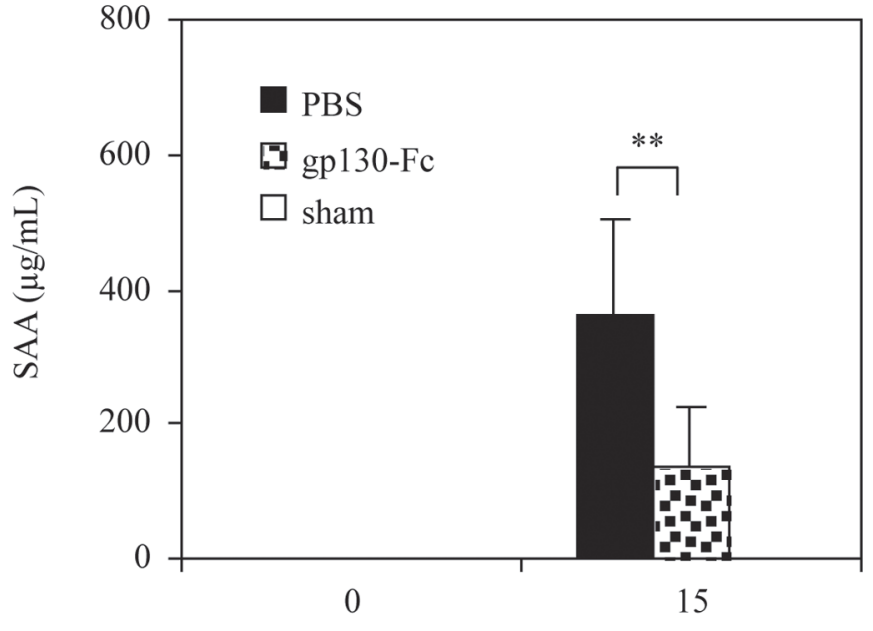

Days after the start of Dp-application

B

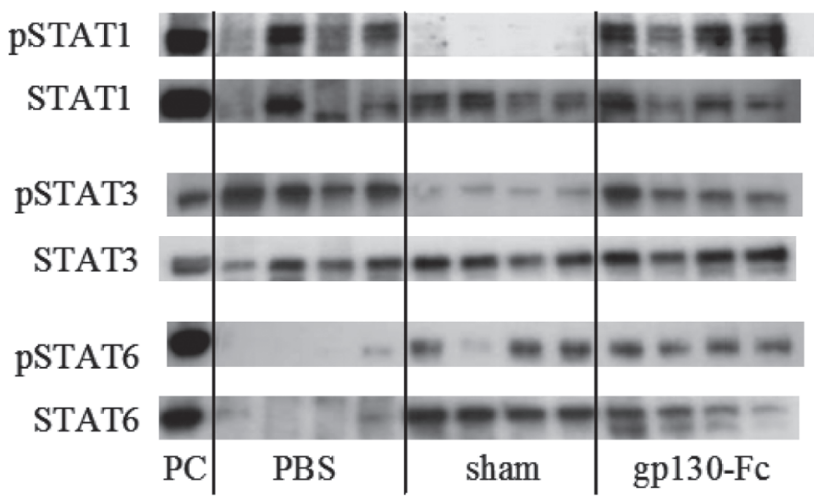

Figure 4. Effect of glycoprotein 130 fused to the Fc region of IgG1(gp130-Fc) on the development of dermatitis in NC/Nga mice. Mice were injected intradermally with gp130-Fc or PBS for $7 \mathrm{~d}$, followed by application with SDS and Dermatophagoides pteronyssinus extract (Dp) for 14 d. Administration with gp130-Fc was continued until the day before the mice were euthanized. (A) Using mice on d 14 or 15 after the beginning of Dp application, dermatitis scores, total IgE serum levels, and serum amyloid A (SAA) levels were evaluated. (B) Using the auricles, phosphorylated signal transducer and activator of transcription 1 (pSTAT1), pSTAT3, and pSTAT6 protein expression levels were examined by Western blotting. (A) Data are presented as mean $\pm \mathrm{SD}$ of 5 mice in each group. ${ }^{* *} P<0.01$ compared with the control group using the Student's $t$-test. (B) Representative data (4 of 5 mice in each group) are shown. Positive control (PC) represents each pSTAT or STAT protein.

We determined whether decreased IL-6 production resulted in a reduction of the severity of dermatitis or merely arose from improved skin inflammation. To this end, we compared immune responses of $\mathrm{NC} / \mathrm{Nga}$ and $\mathrm{BALB} / \mathrm{c}$ mice that were topically administered with Dp. The dermatitis score and SAA level were markedly higher in $\mathrm{NC} / \mathrm{Nga}$ than BALB/c mice, but $\mathrm{IgE}$ levels were significantly lower. These results show that inflammatory responses, such as elevation of SAA levels, are more related to the pathogenesis of dermatitis than
Th2 responses, such as IgE elevation, in this AD model. Among the cytokines tested, only increases in IL-6 secretion by LNC from NC/Nga mice compared with $\mathrm{BALB} / \mathrm{c}$ was detected, even when dermatitis was not induced. On the contrary, more Th2 cytokines, such as IL-4, were produced by LNC from BALB/c mice. These results indicate that IL- 6 , rather than Th2, is involved in the development of dermatitis in this AD model. They also demonstrate that administration of gp130Fc inhibited the development of dermatitis in this $\mathrm{AD}$ 
model, suggesting that the attenuated IL-6 responses were the cause, and not the result of dermatitis inhibition. In contrast, gp130-Fc failed to decrease elevated levels of $\operatorname{IgE}$ in the same mouse, which supports our data suggesting that suppression of $\operatorname{IgE}$ responses is not essential for the inhibition of dermatitis in this AD model. We confirmed that gp130-Fc inhibited STAT3 phosphorylation, which was reflected by decreased responses to IL-6. On the contrary, STAT6 was decreased in the auricles of dermatitis-induced NC/Nga mice; STAT6 is a critical transcriptional factor that regulates IL-4-mediated immune responses. It is likely that IL-4 has little role in the pathogenesis of dermatitis in this AD model. Among the STAT molecules examined, only activation of STAT3 correlated to the severity of dermatitis. These results confirm data suggesting the involvement of IL-6 in the pathogenesis of dermatitis in this murine AD model.

The mechanism by which OLL1073R-1 inhibits IL-6 responses has been unclear. Some reports have proposed the induction of IL-10 responses as a mechanism by which lactobacilli suppress AD-like inflammation, but IL-10 responses were not enhanced by OLL1073R-1 in our study (data not shown). Another report suggested an IL-10-independent mechanism of inhibition of allergic inflammation by parasites. In that study, mesenteric LNC from IL-10 deficient mice infected with the Heligmosomoides polygyrus parasite transferred suppression of allergic inflammation to sensitized hosts, indicating that IL-10 was not the primary modulator of the allergic response (Wilson et al., 2005). It is unclear if OLL1073R-1 partially shares its suppressive mechanism with helminth, as it has not been determined whether inhibition of IL-6 response participates in that IL-10-independent mechanism.

Our results show that OLL1073R-1 prevents skin inflammation in a mouse model of AD. It also suggests that a reduced inflammatory response, as shown by downregulation of IL-6 and SAA levels, may be partly involved in the suppression of dermatitis. This inhibitory activity was dependent upon the bacterial strain used. We also confirmed that IL-6 plays an important role in the development of skin inflammation in this $\mathrm{NC} / \mathrm{Nga}$ AD model. Although the mechanisms involved in inhibition of dermatitis remain unclear, our data shows that OLL1073R-1 may be an attractive agent for preventing inflammatory diseases, such as dermatitis, in the future.

\section{REFERENCES}

Bunikowski, R., K. Gerhold, M. Bräutigam, E. Hamelmann, H. Renz, and U. Wahn. 2001. Effect of low-dose cyclosporin a microemulsion on disease severity, interleukin-6, interleukin- 8 and tumor necrosis factor alpha production in severe pediatric atopic dermatitis. Int. Arch. Allergy Immunol. 125:344-348.

Chambers, R. E., C. W. Hutton, P. A. Dieppe, and J. T. Whicher. 1991. Comparative study of $\mathrm{C}$ reactive protein and serum amyloid A protein in experimental inflammation. Ann. Rheum. Dis. 50:677-679.

Chiba, Y., K. Shida, S. Nagata, M. Wada, L. Bian, C. Wang, T. Shimizu, Y. Yamashiro, J. Kiyoshima-Shibata, M. Nanno, and K. Nomoto. 2010. Well-controlled proinflammatory cytokine responses of Peyer's patch cells to probiotic Lactobacillus casei. Immunology 130:352-362.

Doganci, A., T. Eigenbrod, N. Krug, G. T. De Sanctis, M. Hausding, V. J. Erpenbeck, el-B. Haddad, H. A. Lehr, E. Schmitt, T. Bopp, K. J. Kallen, U. Herz, S. Schmitt, C. Luft, O. Hecht, J. M. Hohlfeld, H. Ito, N. Nishimoto, K. Yoshizaki, T. Kishimoto, S. RoseJohn, H. Renz, M. F. Neurath, P. R. Galle, and S. Finotto. 2005. The IL-6R alpha chain controls lung $\mathrm{CD} 4^{+} \mathrm{CD} 25^{+}$Treg development and function during allergic airway inflammation in vivo. J. Clin. Invest. 115:313-325.

Ganapathi, M. K., L. T. May, D. Schultz, A. Brabenec, J. Weinstein P. B. Sehgal, and I. Kushner. 1988. Role of interleukin-6 in regulating synthesis of C-reactive protein and serum amyloid A in human hepatoma cell lines. Biochem. Biophys. Res. Commun. 157:271-277.

Goodman, W. A., A. D. Levine, J. V. Massari, H. Sugiyama, T. S. McCormick, and K. D. Cooper. 2009. IL-6 signaling in psoriasis prevents immune suppression by regulatory $\mathrm{T}$ cells. J. Immunol. 183:3170-3176.

Han, Y., B. Kim, J. Ban, J. Lee, B. J. Kim, B. S. Choi, S. Hwang, K. Ahn, and J. Kim. 2012. A randomized trial of Lactobacillus plantarum CJLP133 for the treatment of atopic dermatitis. Pediatr. Allergy Immunol. 23:667-673.

Hasegawa, T., K. Hirakawa, T. Matsumoto, S. Toki, Y. Maeyama, and F. Morimatsu. 2009. Efficacy of Lactobacillus plantarum strain HSK201 in relief from Japanese cedar pollinosis. Biosci. Biotechnol. Biochem. 73:2626-2631.

Ishihara, K., and T. Hirano. 2002. IL-6 in autoimmune disease and chronic inflammatory proliferative disease. Cytokine Growth Factor Rev. 13:357-368.

Kalliomäki, M., S. Salminen, H. Arvilommi, P. Kero, P. Koskinen, and E. Isolauri. 2001. Probiotics in primary prevention of atopic disease: A randomised placebo-controlled trial. Lancet 357:10761079.

Kalliomäki, M., S. Salminen, T. Poussa, H. Arvilommi, and E. Isolauri. 2003. Probiotics and prevention of atopic disease: 4-year follow-up of a randomised placebo-controlled trial. Lancet 361:1869-1871.

Kano, H., T. Kaneko, and S. Kaminogawa. 2002a. Oral intake of Lactobacillus delbrueckii ssp. bulgaricus OLL1073R-1 prevents collagen-induced arthritis in mice. J. Food Prot. 65:153-160.

Kano, H., O. Mogami, and M. Uchida. 2002b. Oral administration of milk fermented with Lactobacillus delbrueckii ssp. bulgaricus OLL1073R-1 to DBA/1 mice inhibits secretion of proinflammatory cytokines. Cytotechnology 40:67-73.

Lee, C. E., M. E. Neuland, H. G. Teaford, B. F. Villacis, P. S. Dixon, S. Valtier, C. H. Yeh, D. C. Fournier, and E. N. Charlesworth. 1992. Interleukin- 6 is released in the cutaneous response to allergen challenge in atopic individuals. J. Allergy Clin. Immunol. 89:1010-1020.

Masuda, Y., T. Takahashi, K. Yoshida, Y. Nishitani, M. Mizuno, and H. Mizoguchi. 2012. Anti-allergic effect of lactic acid bacteria isolated from seed mash used for brewing sake is not dependent on the total IgE levels. J. Biosci. Bioeng. 114:292-296.

Park, S. J., T. Nakagawa, H. Kitamura, T. Atsumi, H. Kamon, S. Sawa, D. Kamimura, N. Ueda, Y. Iwakura, K. Ishihara, M. Murakami, and T. Hirano. 2004. IL-6 regulates in vivo dendritic cell differentiation through STAT3 activation. J. Immunol. 173:38443854 .

Rincon, M. 2012. Interleukin-6: From an inflammatory marker to a target for inflammatory diseases. Trends Immunol. 33:571-577.

Samochocki, Z., M. Alifier, P. Bodera, R. Jeziorkowska, E. Rosiak, B. Jurkiewicz, O. Glińska, W. Gliński, and W. Stankiewicz. 2012. T- 
regulatory cells in severe atopic dermatitis: Alterations related to cytokines and other lymphocyte subpopulations. Arch. Dermatol. Res. 304:795-801.

Sano, S., K. S. Chan, S. Carbajal, J. Clifford, M. Peavey, K. Kiguchi, S. Itami, B. J. Nickoloff, and J. DiGiovanni. 2005. Stat3 links activated keratinocytes and immunocytes required for development of psoriasis in a novel transgenic mouse model. Nat. Med. 11:43-49.

Sano, S., K. S. Chan, and J. DiGiovanni. 2008. Impact of Stat3 activation upon skin biology: A dichotomy of its role between homeostasis and diseases. J. Dermatol. Sci. 50:1-14.

Sano, S., S. Itami, K. Takeda, M. Tarutani, Y. Yamaguchi, H. Miura, K. Yoshikawa, S. Akira, and J. Takeda. 1999. Keratinocyte-specific ablation of Stat3 exhibits impaired skin remodeling, but does not affect skin morphogenesis. EMBO J. 18:4657-4668.

Sashihara, T., N. Sueki, and S. Ikegami. 2006. An analysis of the effectiveness of heat-killed lactic acid bacteria in alleviating allergic diseases. J. Dairy Sci. 89:2846-2855.

Sawada, J., H. Morita, A. Tanaka, S. Salminen, F. He, and H. Matsuda. 2007. Ingestion of heat-treated Lactobacillus rhamnosus GG prevents development of atopic dermatitis in NC/Nga mice. Clin. Exp. Allergy 37:296-303.

Shimazu, T., J. Villena, M. Tohno, H. Fujie, S. Hosoya, T. Shimosato, H. Aso, Y. Suda, Y. Kawai, T. Saito, S. Makino, S. Ikegami, H.
Itoh, and H. Kitazawa. 2012. Immunobiotic Lactobacillus jensenii elicits anti-inflammatory activity in porcine intestinal epithelial cells by modulating negative regulators of the Toll-like receptor signaling pathway. Infect. Immun. 80:276-288.

Shimizu, N., K. Dairiki, S. Ogawa, and T. Kaneko. 2006. Dietary whey protein hydrolysate suppresses development of atopic dermatitislike skin lesions induced by mite antigen in NC/Nga mice. Allergol. Int. 55:185-189.

Spergel, J. M., E. Mizoguchi, H. Oettgen, A. K. Bhan, and R. S. Geha. 1999. Roles of $T_{H} 1$ and $T_{H} 2$ cytokines in a murine model of allergic dermatitis. J. Clin. Invest. 103:1103-1111.

Unno, T., H. Suto, T. Yoshiike, H. Ogawa, and C. Ra. 2001. Induction of atopic dermatitis-like skin lesion in NC/Nga mice-The influence of the skin barrier destroying solution to the induction of dermatitis. Arerugi 50:1152-1162. (in Japanese).

Wilson, M. S., M. D. Taylor, A. Balic, C. A. Finney, J. R. Lamb, and R. M. Maizels. 2005. Suppression of allergic airway inflammation by helminth-induced regulatory T cells. J. Exp. Med. 202:11991212

Wüthrich, B. 1978. Serum IgE in atopic dermatitis: Relationship to severity of cutaneous involvement and course of disease as well as coexistence of atopic respiratory diseases. Clin. Allergy 8:241-248. 